\title{
Method of automorphic functions for an inverse problem of antiplane elasticity
}

\author{
Y.A. Antipov \\ Department of Mathematics, Louisiana State University \\ Baton Rouge LA 70803, USA
}

\begin{abstract}
A nonlinear inverse problem of antiplane elasticity for a multiply connected domain is examined. It is required to determine the profile of $n$ uniformly stressed inclusions when the surrounding infinite body is subjected to antiplane uniform shear at infinity. A method of conformal mappings of circular multiply connected domains is employed. The conformal map is recovered by solving consequently two RiemannHilbert problems for piecewise analytic symmetric automorphic functions. For domains associated with the first class Schottky groups a series-form representation of a $(3 n-4)$-parametric family of conformal maps solving the problem is discovered. Numerical results for two and three uniformly stressed inclusions are reported and discussed.
\end{abstract}

\section{Introduction}

Considerable interest in inverse boundary value problems for partial differential equations has developed since the work by Riabuchinsky (1) on recovering the boundary of a domain when a function is harmonic inside and the function and its normal derivative are prescribed in the boundary. Numerous applications of inverse problems to filtration and hydroaerodynamics advanced the development of a qualitative theory and various constructive techniques including those based on the theory of boundary value problems of the theory of analytic functions (2). The classical work by Eshelby (3) on determination of the shapes of curvilinear cavities and inclusions with prescribed properties inspired material scientists to work on inverse problems of elasticity. Eshelby found that if the unbounded elastic body is uniformly loaded at infinity and the body has an elliptic or ellipsoidal inclusion with different elastic constants, then the stress field is uniform inside the inclusion. Eshelby's conjecture that in plane and antiplane cases there are no other shapes apart from ellipses was proved in (4). Another proof based on the method of conformal mappings was later proposed in (5).

An inverse problem of plane elasticity for a plane uniformly loaded at infinity and having $n$ holes was examined by Cherepanov ([6). In this model, the holes boundaries are subjected to constant normal and tangential traction, and the boundaries have to be determined from the condition that the tangential normal stress is constant in all the contours. Cherepanov employed the method of conformal mappings and homogeneous Schwarz problems to recover the shapes of two symmetric holes. A circular map from the exterior of $n$-circles onto the $n$-connected elastic domain, integral equations, and the method of least squares for their numerical solution was proposed in (7). An explicit representation in terms of the Weierstrass elliptic function for the profile of an inclusion in the case of a doubly periodic structure was found in (8). Recently, the theory of the 
Cherepanov problem for $n$ inclusions was advanced in (9) by developing a method of the Riemann-Hilbert problem on a Riemann surface. It was shown that for any $n \geq 1$ there always exists a set of the loading parameters which generate inadmissible poles of the solution.

For the antiplane inverse problem on reconstructing the boundaries of two symmetric uniformly stressed inclusions the Weierstrass zeta function and the Schwarz-Christoffel formula were found to be effective in (10). A method of Laurent series and a conformal mapping from an annulus to a doubly connected domain to recover the profile of two inclusions with uniform stresses was applied in (11). Different numerical approaches for inverse antiplane problems were employed in (12), (13).

Two methods for nonlinear inverse problems on supercavitating flow past $n$ hydrofoils were proposed in (14), (15). Both methods are based on the existence theorem (16), (17) of a conformal map of an $n$ connected parametric slit or circular domain into the $n$-connected physical domain. These methods express the conformal map in terms of the solutions of two Riemann-Hilbert problems in a multiply connected canonical domain. In the first method, the Riemann-Hilbert problems are set in $n$ slits and reduce to two Riemann-Hilbert problems on a genus- $n$ Riemann surface. The parametric domain for the second technique is the exterior of $n$ circles. The method employs linear fractional transformations, a symmetry transformation, and the Schottky groups (18) and leads to two Riemann-Hilbert problems of the theory of symmetric automorphic functions (19), (20), (21) (15), (22). Alternatively, the problems could be solved by using the theory of

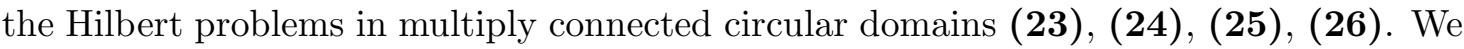
also note that numerous conformal maps of $n$-connected canonical domains into physical domains exists in the literature when the boundary conditions of the problem allows for bypassing the Hilbert problem in the circular domain. Examples include a map from a multiply connected circular domain into a multiply connected polygonal region in a series form (27) and in terms of the Schottky-Klein prime function of the Schottky group associated with the circular domain (28).

In this paper we aim to propose an exact method of conformal mappings and the Riemann-Hilbert problem of the theory of automorphic functions for the inverse antiplane problem on $n$ inclusions. The inclusions may have different shear moduli and are in ideal contact with the surrounding elastic matrix subjected at infinity to uniform antiplane shear $\tau_{13}=\tau_{1}^{\infty}$ and $\tau_{23}=\tau_{2}^{\infty}$. The profiles of the inclusions are not prescribed and have to be determined from the condition that the stress field inside all the inclusions is uniform, $\tau_{13}=\tau_{1}$ and $\tau_{23}=\tau_{2}$.

In Section 2, we formulate the problem, map the exterior of $n$ circles into the exterior of $n$ uniformly stressed inclusions and reduce the problem of determination of the conformal map to two inhomogeneous Schwarz problems to be solved consecutively. In Section 3, we convert the Schwarz problems to two Riemann-Hilbert problems for piecewise analytic symmetric automorphic functions. For their solution we employ a qusiautomorphic analogue of the Cauchy kernel (19), (21). Note that the Cauchy kernel analogue (29) in terms of the Schottky-Klein prime function of the Schottky group could also be employed. In Section 4, for the first class Schottky groups (30), we write down a series representation of a family of conformal maps solving the problem. The family has $3 n-4$ free parameters and should satisfy the natural restriction that the inclusions contours cannot overlap. We also give some sample profiles of two and three uniformly stressed inclusions. In Appendix, for completeness, we examine the case $n=1$ and show that the profile of a single uniformly stressed inclusion is an ellipse. 


\section{Formulation}

Consider the following problem of antiplane elasticity.

Let $D_{0}, D_{1}, \ldots, D_{n-1}$ be $n$ finite inclusions in an infinite isotropic solid. The shear moduli of the inclusions and the solid $D^{e}=\mathbb{R}^{2} \backslash D\left(D=\cup_{j=0}^{n-1} D_{j}\right)$ are taken to be $\mu_{j}$ and $\mu$, respectively. It is assumed that the inclusions are in ideal contact with the matrix, and the whole solid $D^{e} \cup D$ is in a state of antiplane shear due to constant shear stresses applied at infinity, $\tau_{13}=\tau_{1}^{\infty}, \tau_{23}=\tau_{2}^{\infty}$. It is aimed to determine the boundaries of the inclusions, $L_{j}$, such that the stresses $\tau_{13}$ and $\tau_{23}$ are constant in all the inclusions $D_{j}$, $\tau_{13}=\tau_{1}, \tau_{23}=\tau_{2}, j=0,1, \ldots, n-1$.

Let $u$ and $u_{j}$ be the $x_{3}$-components of the displacement vectors for the body $D^{e}$ and the inclusions $D_{j}$, respectiely. Then $\tau_{k 3}=\mu \partial u / \partial x_{k}(k=1,2),\left(x_{1}, x_{2}\right) \in D^{e}$, and $\tau_{k 3}=\mu_{j} \partial u / \partial x_{k}(k=1,2),\left(x_{1}, x_{2}\right) \in D_{j}, j=0,1, \ldots, n-1$. At infinity, the displacement $u$ is growing as

$$
u \sim \mu^{-1}\left(\tau_{1}^{\infty} x_{1}+\tau_{2}^{\infty} x_{2}\right)+\text { const }, \quad x_{1}^{2}+x_{2}^{2} \rightarrow \infty .
$$

Due to the fact that the stresses $\tau_{12}$ and $\tau_{13}$ are constant in the inclusions, the $x_{3^{-}}$ displacements $u_{j}$ for $\left(x_{1}, x_{2}\right) \in D_{j}$ are linear functions

$$
u_{j}=\mu_{j}^{-1}\left(\tau_{1} x_{1}+\tau_{2} x_{2}\right)+d_{j}^{\prime}, \quad\left(x_{1}, x_{2}\right) \in D_{j}, \quad j=0,1, \ldots, n-1,
$$

and $d_{j}^{\prime}$ are real constants.

Let $v$ and $v_{j}$ be the harmonic conjugates of the harmonic functions $u$ and $u_{j}$ in the domains $D^{e}$ and $D_{j}$, respectively. Denote $z=x_{1}+i x_{2}$. Then $\phi(z)=u\left(x_{1}, x_{2}\right)+i v\left(x_{1}, x_{2}\right)$ and $\phi_{j}(z)=u_{j}\left(x_{1}, x_{2}\right)+i v_{j}\left(x_{1}, x_{2}\right)$ are analytic functions in the corresponding domains $D^{e}$ and $D_{j}$. The boundary conditions of ideal contact imply that the traction component $\tau_{\nu 3}$ and the $x_{3}$-component of the displacement are continuous through the contours $L_{j}$,

$$
\mu \frac{\partial u}{\partial \nu}=\mu_{j} \frac{\partial u_{j}}{\partial \nu}, \quad u=u_{j}, \quad\left(x_{1}, x_{2}\right) \in L_{j}, \quad j=0,1, \ldots, n-1,
$$

where $\frac{\partial}{\partial \nu}$ is the normal derivative. In terms of the functions $\phi_{j}(z)$ the ideal contact boundary conditions can be written as

$$
\frac{\kappa_{j}+1}{2} \phi_{j}(z)-\frac{\kappa_{j}-1}{2} \overline{\phi_{j}(z)}=\phi(z)+i b_{j}, \quad z \in L_{j}, \quad j=0,1, \ldots, n-1,
$$

where $\kappa_{j}=\mu_{j} / \mu$, and $b_{j}$ are real constants. The equivalence of the boundary conditions (2.3) and (2.4) follows from the Cauchy-Riemann condition $\frac{\partial u}{\partial \nu}=\frac{\partial v}{\partial s}$, where $\frac{\partial}{\partial s}$ is the tangential derivative. Since the functions $u_{j}$ are known everywhere in the domains $D_{j}$, the functions $\phi_{j}(z)$ are defined up to arbitrary constants and given by by

$$
\phi_{j}(z)=\frac{\bar{\tau} z}{\mu_{j}}+d_{j}, \quad z \in D_{j}, \quad j=0,1, \ldots, n-1,
$$

where $\bar{\tau}=\tau_{1}-i \tau_{2}, d_{j}=d_{j}^{\prime}+i d_{j}^{\prime \prime}, d_{j}^{\prime \prime}$ are real constants. In view of the relations (2.5), instead of the function $\phi(z)$, it is convenient to deal with the function $f(z)=\phi(z)-\bar{\tau} z / \mu$, $z \in D^{e}$. The new function $f(z)$ is analytic in the domain $D^{e}$, satisfies the boundary condition

$$
f(z)=\frac{1}{\lambda_{j}} \operatorname{Re}\left(\frac{\bar{\tau}}{\mu} z\right)+d_{j}^{\prime}+i a_{j}, \quad z \in L_{j}, \quad j=0,1, \ldots, n-1
$$


and the condition at infinity

$$
f(z) \sim \frac{\left(\bar{\tau}^{\infty}-\bar{\tau}\right) z}{\mu}+\text { const }, \quad z \rightarrow \infty .
$$

Here, $\lambda_{j}=\kappa_{j} /\left(1-\kappa_{j}\right), a_{j}=\kappa_{j} d_{j}^{\prime \prime}-b_{j}$ are real constants, and $\bar{\tau}^{\infty}=\tau_{1}^{\infty}-i \tau_{2}^{\infty}$. The condition (2.7) is due to the relation $\phi(z) \sim \bar{\tau}^{\infty} z / \mu+$ const, $z \rightarrow \infty$.

Let $z=\omega(\zeta)$ be a conformal map that transforms the exterior of $n$ circles $\mathfrak{L}_{j}(j=$ $0,1, \ldots, n-1)$ into the physical domain $D^{e}$. Denote this $n$-connected circular domain by $\mathfrak{D}^{e}$. By scaling and rotation, it is always possible to achieve (16), (17) that one of the circles say, $\mathfrak{L}_{0}$, is of unit radius and centered at the origin and, in addition, the center of another circle say, $\mathfrak{L}_{1}$, falls in the real axis. Let the circular map meet the condition $\omega(\infty)=\infty$. In this case, if the original problem has a unique solution, then the radius of $\mathfrak{L}_{1}$, the complex centers and the radii of the rest $n-2$ circles cannot be selected arbitrarily.

In the vicinity of the infinite point the conformal map can be represented as

$$
\omega(\zeta)=c_{-1} \zeta+c_{0}+\sum_{j=1}^{\infty} \frac{c_{j}}{\zeta^{j}},
$$

where $c_{-1}=c_{-1}^{\prime}+i c_{-1}^{\prime \prime}$. Denote $f(\omega(\zeta))=F(\zeta)$. From the boundary condition (2.6) we deduce that the functions $F(\zeta)$ and $\omega(\zeta)$ satisfy the following two Schwarz problems to be solved consecutively.

Find two functions $F(\zeta)$ and $\omega(\zeta)$ analytic in the domain $\mathfrak{D}^{e}$ and continuous up to the boundary $\mathfrak{L}=\cup_{j=0}^{n-1} \mathfrak{L}_{j}$ such that

$$
\operatorname{Im} F(\zeta)=a_{j}, \quad \zeta \in \mathfrak{L}_{j}, \quad j=0,1 \ldots, n-1,
$$

and

$$
\operatorname{Re}\left[\frac{\bar{\tau}}{\mu} \omega(\zeta)\right]=\lambda_{j}\left[\operatorname{Re} F(\zeta)-d_{j}^{\prime}\right], \quad \zeta \in \mathfrak{L}_{j}, \quad j=0,1 \ldots, n-1 .
$$

At the infinite point, both of the functions have a simple pole,

$$
F(\zeta) \sim \frac{\bar{\tau}^{\infty}-\bar{\tau}}{\mu} c_{-1} \zeta, \quad \omega(\zeta) \sim c_{-1} \zeta, \quad \zeta \rightarrow \infty
$$

In addition, the function $\omega: \mathfrak{L}_{j} \rightarrow L_{j}(j=0, \ldots, n-1)$ has to be univalent, and the interiors of the images of the contours $\mathfrak{L}_{j}$, the domains $D_{j}$, are disjoint sets.

\section{Riemann-Hilbert problems of the theory of automorphic functions}

\subsection{Setting}

Let $\mathfrak{G}$ be the symmetry group of the circular line $\mathfrak{L}=\mathfrak{L}_{0} \cup \ldots \cup \mathfrak{L}_{n-1}$ generated by the linear transformations $\sigma_{j}=T_{j} T_{0}(\zeta), j=0,1, \ldots, n-1$, where $T_{j}$ are linear fractional transformations

$$
T_{j}(\zeta)=\zeta_{j}+\frac{r_{j}^{2}}{\bar{\zeta}-\bar{\zeta}_{j}}, \quad j=0,1, \ldots, n-1 .
$$

Here, $r_{j}$ and $\zeta_{j}$ are the radius and the center of the circle $\mathfrak{L}_{j}$. The transformation $\sigma_{j}(\zeta)$ $(j=1, \ldots, n-1)$ maps the exterior domain $\mathfrak{D}^{e}$ into the exterior of $\sigma_{j}\left(\mathfrak{L}_{m}\right) \subset$ int $\mathfrak{L}_{j}$, $j, m=1, \ldots, n-1$. Denote by $\tilde{\mathfrak{D}}^{e}=T_{0}\left(\mathfrak{D}^{e}\right)$ the exterior of the circles $T_{0}\left(\mathfrak{L}_{j}\right)$ inside $\mathfrak{L}_{0}$. 
The domains $\mathfrak{D}^{e}$ and $\tilde{\mathfrak{D}}^{e}$ and the contour $\mathfrak{L}$ comprises a fundamental region $\mathfrak{F}_{\mathfrak{G}}$ of the group $\mathfrak{G}: \mathfrak{F}_{\mathfrak{G}}=\mathfrak{D} \cup \tilde{\mathfrak{D}}^{e} \cup \mathfrak{L}$. The group $\mathfrak{G}$ is a symmetry Schottky group (18). It consists of the identical map $\sigma_{0}(\zeta)=T_{0} T_{0}(\zeta)=\zeta$ and all possible compositions of the generators $\sigma_{j}$ and the inverse maps $\sigma_{j}^{-1}=T_{0} T_{j} j=1,2, \ldots, n-1$. Therefore, each element of the group $\mathfrak{G}$ is a composition of an even number of the symmetry maps $T_{j}(\zeta)(j=0,1, \ldots, n-1)$,

$$
\begin{gathered}
\sigma=T_{k_{1}} T_{k_{2}} \ldots T_{k_{2 s-1}} T_{k_{2 s}}, \\
k_{2} \neq k_{1}, k_{3} \neq k_{2}, \ldots, k_{2 s} \neq k_{2 s-1}, \quad k_{1}, k_{2}, \ldots k_{2 s}=0,1, \ldots, n-1 .
\end{gathered}
$$

The region $\Omega=\cup_{\sigma \in \mathfrak{G}} \sigma\left(\mathfrak{F}_{\mathfrak{G}}\right)$ is invariant with respect to the group $\mathfrak{G}: \sigma(\Omega)=\Omega$ for any $\sigma \in \mathfrak{G}$, where $\Omega=\overline{\mathbb{C}} \backslash \Lambda, \overline{\mathbb{C}}=\mathbb{C} \cup\{\infty\}$, and $\Lambda$ is the set of all limit points of the group $\mathfrak{G}$. If $n=2$, then the set $\Lambda$ consists of two points, while for $n \geq 3$, the number of limit points is infinite. All maps of the group $\mathfrak{G}$ are linear fractional transformations

$$
\sigma(\zeta)=\frac{\alpha_{\sigma} \zeta+\beta_{\sigma}}{\gamma_{\sigma} \zeta+\delta_{\sigma}}, \quad \alpha_{\sigma} \delta_{\sigma}-\gamma_{\sigma} \beta_{\sigma} \neq 0
$$

and $\gamma_{\sigma} \neq 0$ if $\sigma \neq \sigma_{0}$. It is assumed that the series

$$
\sum_{\sigma \in \mathfrak{G} \backslash \sigma_{0}} \frac{\left|\alpha_{\sigma} \delta_{\sigma}-\beta_{\sigma} \gamma_{\sigma}\right|}{\left|\gamma_{\sigma}\right|^{2}}
$$

is convergent. If $\mathfrak{G}$ is a first class group (30), that is if the condition (3.4) is satisfied, then each $\mathfrak{G}$-automorphic function is representable as a series with simple fractions as its elements. By the sufficient Schottky condition (131) $\mathfrak{G}$ is a first class group if the domain $\mathfrak{D}$ can be split into a union of triply or doubly connected domains by circles which do not intersect each other and the circles $\mathfrak{L}_{j}(j=0,1, \ldots, n-1)$. Remark that $\mathfrak{G}$ is a first class Schottky group if it is associated with a doubly or triply connected domains, or a circular multiply connected domain when the centers of the circles $\mathfrak{L}_{j}$ lie in a straight line, or it satisfies the Aksentiev condition (23). An example of the domain $\mathfrak{D}$ that generates a symmetry Schottky group for which the series (3.5) is divergent and the corresponding Poincaré series of dimension -2 is not absolutely convergent is given in (21). In what follows, it is assumed that the domain $\mathfrak{D}$ obeys the sufficient conditions which guarantee the convergence of the series (3.4). This justifies the change of order of summation used in the representation of a quasiautomorphic analogue of the Cauchy kernel.

Introduce next two functions, $\Phi_{1}(\zeta)$ and $\Phi_{2}(\zeta)$, analytic in the domain $\mathfrak{D}^{e}$ by

$$
\Phi_{1}(\zeta)=F(\zeta)-c \zeta, \quad \Phi_{2}(\zeta)=\frac{i \bar{\tau}}{\mu}\left[\omega(\zeta)-c_{-1} \zeta\right], \quad \zeta \in \mathfrak{D}^{e}
$$

where

$$
c=\frac{\bar{\tau}^{\infty}-\bar{\tau}}{\mu} c_{-1}
$$

To extend their definition inside the domain $\mathfrak{D}$, we set

$$
\begin{gathered}
\Phi_{m}(\zeta)=\overline{\Phi_{m}\left(T_{0}(\zeta)\right)}, \quad \sigma \in T_{0}\left(\mathfrak{D}^{e}\right), \\
\Phi_{m}(\zeta)=\Phi_{m}\left(\sigma^{-1}(\zeta)\right), \quad \zeta \in \sigma\left(\mathfrak{D}^{e} \cup T_{0}\left(\mathfrak{D}^{e}\right)\right), \quad \sigma \in \mathfrak{G} .
\end{gathered}
$$

Then $\Phi_{m}(\zeta)$ are piecewise meromorphic and $\mathfrak{G}$-automorphic functions which satisfy the symmetry condition

$$
\overline{\Phi_{m}\left(T_{j}(\zeta)\right)}=\Phi_{m}(\zeta), \quad \zeta \in T_{j}\left(\mathfrak{D}^{e}\right)=\sigma_{j}\left(T_{0}\left(\mathfrak{D}^{e}\right)\right), \quad j=1,2, \ldots n-1
$$


All circles $\sigma(\mathfrak{L})$ including $\mathfrak{L}$ are discontinuity lines for the functions $\Phi_{m}(\zeta)$. Let $\Phi_{m}^{+}(\xi)$ and $\Phi_{m}^{-}(\xi)$ be the boundary values of the functions $\Phi_{m}(\zeta)$ from the interior and the exterior of the circles $\sigma(\mathfrak{L}), \sigma \in \mathfrak{G}$, respectively. Then the functions $\Phi_{m}(\zeta)$ solve the following two Riemann-Hilbert problems.

Find all piecewise analytic and $\mathfrak{G}$-automorphic functions bounded at infinity which meet the symmetry condition (3.8) and satisfy the linear relation in the circles $\mathfrak{L}_{j}$

$$
\Phi_{m}^{+}(\xi)-\Phi_{m}^{-}(\xi)=g_{m j}(\xi), \quad \xi \in \mathfrak{L}_{j}, \quad j=0,1, \ldots, n-1, \quad m=1,2,
$$

where

$$
g_{1 j}(\xi)=2 i\left[\operatorname{Im}(c \xi)-a_{j}\right], \quad g_{2 j}(\xi)=-2 i \operatorname{Re}\left\{\frac{\kappa_{j}}{1-\kappa_{j}}\left[F(\xi)-d_{j}^{\prime}\right]-\frac{\bar{\tau}}{\mu} c_{-1} \xi\right\} .
$$

Due to formulas (3.5) to determine the conformal mapping $\omega(\zeta)$, it is required to find the function $\Phi_{2}(\zeta)$. This can be done only if the first function $\Phi_{1}(\zeta)$ is known. To solve the two Riemann-Hilbert problems (3.9), we employ the following singular integral:

$$
\Psi(\zeta)=\frac{1}{2 \pi i} \int_{\mathfrak{L}} \mathcal{K}(\zeta, \eta) g(\eta) d \eta
$$

where $\mathcal{K}(\zeta, \eta)$ is the series (19), (21)

$$
\mathcal{K}(\zeta, \eta)=\sum_{\sigma \in \mathfrak{G}}\left(\frac{1}{\sigma(\eta)-\zeta}-\frac{1}{\sigma(\eta)-\zeta_{*}}\right) \sigma^{\prime}(\eta)
$$

$\zeta_{*} \in \mathfrak{D}^{e}$ is an arbitrary fixed point, and $g(\eta)$ is a density. Alternatively, because of the identity

$$
\left(\frac{1}{\sigma(\eta)-\zeta}-\frac{1}{\sigma(\eta)-\zeta_{*}}\right) \sigma^{\prime}(\eta)=\frac{1}{\eta-\sigma^{-1}(\zeta)}-\frac{1}{\eta-\sigma^{-1}\left(\zeta_{*}\right)}
$$

the kernel $\mathcal{K}(\zeta, \eta)$ can be written as

$$
\mathcal{K}(\zeta, \eta)=\sum_{\omega \in \mathfrak{G}}\left(\frac{1}{\eta-\omega(\zeta)}-\frac{1}{\eta-\omega\left(\zeta_{*}\right)}\right) .
$$

Here, since $\sigma \in \mathfrak{G}$ implies $\sigma^{-1} \in \mathfrak{G}$, we made the substitution $\omega=\sigma^{-1}$. The absolute convergence of the series in (3.13) and (3.14) is guaranteed by the convergence of the series (3.4). This circumstance allows for changing the order of summation and integration in the expression (3.11). Since the identity transformation $\sigma_{0} \in \mathfrak{G}$, the series admits the representation

$$
\mathcal{K}(\zeta, \eta)=\frac{1}{\eta-\zeta}+\mathcal{K}_{0}(\zeta, \eta)
$$

where $\mathcal{K}_{0}(\zeta, \eta)$ is an analytic function of $\zeta$ in the domain $\Omega$

$$
\mathcal{K}_{0}(\zeta, \eta)=-\frac{1}{\eta-\zeta_{*}}+\sum_{\sigma \in \mathfrak{G} \backslash \sigma_{0}}\left(\frac{1}{\eta-\sigma(\zeta)}-\frac{1}{\eta-\sigma\left(\zeta_{*}\right)}\right)
$$

For our next step, we need to use the following property of the kernel $\mathcal{K}(\zeta, \eta)(\mathbf{1 9})$, (21):

$$
\mathcal{K}(\sigma(\zeta), \eta)=\mathcal{K}(\zeta, \eta)+\chi_{\sigma}(\eta), \quad \sigma \in \mathfrak{G},
$$

where

$$
\chi_{\sigma}(\eta)=\mathcal{K}\left(\sigma\left(\zeta_{*}\right), \eta\right) .
$$

The two relations (3.15) and (3.17) classify the function $\mathcal{K}(\zeta, \eta)$ as a quasiauthomorphic analogue of the Cauchy kernel. 


\subsection{Solution $\Phi_{1}(\zeta)$ of the first Riemann-Hilbert problem}

We next claim that under a certain choice of the real constants $a_{j}$ the function

$$
\Phi_{1}(\zeta)=\Psi_{1}(\zeta)+\overline{\Psi_{1}\left(T_{0}(\zeta)\right)}+C_{1},
$$

provides the general solution of the first Riemann-Hilbert problem (3.9). Here, $C_{1}$ is an arbitrary real constant and

$$
\Psi_{1}(\zeta)=\frac{1}{2 \pi} \sum_{j=0}^{n-1} \int_{\mathfrak{L}_{j}}\left[\operatorname{Im}(c \eta)-a_{j}\right] \mathcal{K}(\zeta, \eta) d \eta
$$

Apparently, the function $\Phi_{1}(\zeta)$ satisfies the symmetry condition $\Phi_{1}(\zeta)=\overline{\Phi_{1}\left(T_{0}(\zeta)\right)}, \sigma \in$ $T_{0}\left(\mathfrak{D}^{e}\right)$. Because of the quasiautomorphicity of the kernel $\mathcal{K}(\zeta, \eta)$, in general, the function $\Phi_{1}(\zeta)$ is not automorphic. However, by a certain choice of the constants $a_{j}$, it is possible to satisfy the condition $\Phi_{1}(\zeta)=\Phi_{1}\left(\sigma_{j}(\zeta)\right), j=1,2, \ldots, n-1$, and make the solution automorphic. Indeed, the relation (3.17) implies

$$
\mathcal{K}\left(\sigma_{j}(\zeta), \eta\right)=\mathcal{K}(\zeta, \eta)+\chi_{\sigma_{j}}(\eta), \quad \chi_{\sigma_{j}}(\eta)=\mathcal{K}\left(\sigma_{j}\left(\zeta_{*}\right), \eta\right), \quad j=1,2, \ldots, n-1 .
$$

Hence

$$
\Psi_{1}\left(\sigma_{j}(\zeta)\right)=\Psi_{1}(\zeta)+e_{j}, \quad j=1,2, \ldots, n-1
$$

where

$$
e_{j}=\frac{1}{2 \pi} \sum_{l=0}^{n-1} \int_{\mathfrak{L}_{l}}\left[\operatorname{Im}(c \eta)-a_{l}\right] \chi_{\sigma_{j}}(\eta) d \eta
$$

On writing the relation (3.22) as $\Psi_{1}(\zeta)=\Psi_{1}\left(\sigma_{j}^{-1}(\zeta)\right)+e_{j}, j=1,2, \ldots, n-1$, there is no difficulty in verifying that

$$
\overline{\Psi_{1}\left(T_{0} \sigma_{j}(\zeta)\right)}=\overline{\Psi_{1}\left(\sigma_{j}^{-1} T_{0}(\zeta)\right)}=\overline{\Psi_{1}\left(T_{0}(\zeta)\right)}-\overline{e_{j}} .
$$

In view of (3.22) and (3.24) we obtain

$$
\Phi_{1}\left(\sigma_{j}(\zeta)\right)=\Phi_{1}(\zeta)+e_{j}-\overline{e_{j}}, \quad j=1,2, \ldots, n-1 .
$$

Hence the solution $\Phi_{1}(\zeta)$ is a $\mathfrak{G}$-automorphic function if and only if $\operatorname{Im} e_{j}=0, j=$ $1,2, \ldots, n-1$, that is

$$
\operatorname{Im}\left\{\sum_{l=0}^{n-1} \int_{\mathfrak{L}_{l}}\left[a_{l}-\operatorname{Im}(c \eta)\right] \chi_{\sigma_{j}}(\eta) d \eta\right\}=0, \quad j=1,2, \ldots, n-1 .
$$

The integrals

$$
\int_{\mathfrak{L}_{l}} \chi_{\sigma_{j}}(\eta) d \eta=\sum_{\sigma \in \mathfrak{G}} \int_{\mathfrak{L}_{l}}\left(\frac{1}{\eta-\sigma \sigma_{j}\left(\zeta_{*}\right)}-\frac{1}{\eta-\sigma\left(\zeta_{*}\right)}\right) d \eta, \quad j=1,2, \ldots, n-1,
$$

can be evaluated by the theory of residues. Assume first that $l=0$. If $\sigma=\sigma_{0}$, then $\sigma \sigma_{j}\left(\zeta_{*}\right) \in \operatorname{int} \mathfrak{L}_{j}(j=1,2, \ldots, n-1)$ and $\sigma\left(\zeta_{*}\right)=\zeta_{*} \in \mathfrak{D}^{e}$. Hence,

$$
\int_{\mathfrak{L}_{0}}\left(\frac{1}{\eta-\sigma \sigma_{j}\left(\zeta_{*}\right)}-\frac{1}{\eta-\sigma\left(\zeta_{*}\right)}\right) d \eta=0
$$


If $\sigma=\sigma_{j}^{-1}$ and since $\sigma_{j}^{-1}=T_{0} T_{j}$, then $\sigma \sigma_{j}\left(\zeta_{*}\right) \in \mathfrak{D}^{e}$ and $\sigma\left(\zeta_{*}\right)=T_{0} T_{j}\left(\zeta_{*}\right) \in \operatorname{int} \mathfrak{L}_{0}$. Thus,

$$
\int_{\mathfrak{L}_{0}}\left(\frac{1}{\eta-\sigma \sigma_{j}\left(\zeta_{*}\right)}-\frac{1}{\eta-\sigma\left(\zeta_{*}\right)}\right) d \eta=-2 \pi i .
$$

Let now $\sigma \neq \sigma_{0}, \sigma \neq \sigma_{j}^{-1}$, and $\sigma=T_{k} \ldots T_{\nu}$. If $k \neq 0$, then

$$
\int_{\mathfrak{L}_{0}} \frac{d \eta}{\eta-\sigma \sigma_{j}\left(\zeta_{*}\right)}=\int_{\mathfrak{L}_{0}} \frac{d \eta}{\eta-\sigma\left(\zeta_{*}\right)}=0
$$

In the case $k=0$ we have $\sigma \sigma_{j}\left(\zeta_{*}\right) \in \operatorname{int} \mathfrak{L}_{0}$ and $\sigma\left(\zeta_{*}\right) \in \operatorname{int} \mathfrak{L}_{0}$. That is why

$$
\int_{\mathfrak{L}_{0}} \frac{d \eta}{\eta-\sigma \sigma_{j}\left(\zeta_{*}\right)}=\int_{\mathfrak{L}_{0}} \frac{d \eta}{\eta-\sigma_{j}\left(\zeta_{*}\right)}=2 \pi i
$$

Summing up the results obtained we evaluate the integral (3.27) for $l=0$

$$
\int_{\mathfrak{L}_{0}} \chi_{\sigma_{j}}(\eta) d \eta=-2 \pi i \quad j=1,2, \ldots, n-1 .
$$

Assume next that $l=1,2, \ldots, n-1$ and evaluate the integrals (3.27). If $\sigma=\sigma_{0}$, then $\sigma \sigma_{j}\left(\zeta_{*}\right) \in \operatorname{int} \mathfrak{L}_{j}$, while $\sigma\left(\zeta_{*}\right)=\zeta_{*} \notin$ int $\mathfrak{L}_{j}$. This implies

$$
\int_{\mathfrak{L}_{l}} \frac{d \eta}{\eta-\sigma \sigma_{j}\left(\zeta_{*}\right)}=\left\{\begin{array}{cl}
2 \pi i, & j=l, \\
0, & j \neq l,
\end{array} \int_{\mathfrak{L}_{l}} \frac{d \eta}{\eta-\sigma_{j}\left(\zeta_{*}\right)}=0, \quad l=1,2, \ldots, n-1 .\right.
$$

In the case $\sigma=\sigma_{j}^{-1}$ we have $\sigma \sigma_{j}\left(\zeta_{*}\right)=\zeta_{*} \in \mathfrak{D}^{e}$ and $\sigma\left(\zeta_{*}\right) \in$ int $\mathfrak{L}_{0}$, and therefore

$$
\int_{\mathfrak{L}_{l}} \frac{d \eta}{\eta-\sigma \sigma_{j}\left(\zeta_{*}\right)}=\int_{\mathfrak{L}_{l}} \frac{d \eta}{\eta-\sigma\left(\zeta_{*}\right)}=0
$$

Suppose $\sigma \neq \sigma_{0}, \sigma \neq \sigma_{j}^{-1}$, and $\sigma=T_{l} \ldots T_{\nu}$. This implies that $\left.\sigma \sigma_{j}\left(\zeta_{*}\right)\right) \in$ int $\mathfrak{L}_{l}$ and $\sigma\left(\zeta_{*}\right) \in$ int $\mathfrak{L}_{l}$. Therefore

$$
\int_{\mathfrak{L}_{l}} \frac{d \eta}{\eta-\sigma \sigma_{j}\left(\zeta_{*}\right)}=\int_{\mathfrak{L}_{l}} \frac{d \eta}{\eta-\sigma\left(\zeta_{*}\right)}=2 \pi i .
$$

If $\sigma$ takes on the value $T_{k} \ldots T_{\nu}$ and $k \neq l$, then

$$
\int_{\mathfrak{L}_{l}} \frac{d \eta}{\eta-\sigma \sigma_{j}\left(\zeta_{*}\right)}=\int_{\mathfrak{L}_{l}} \frac{d \eta}{\eta-\sigma\left(\zeta_{*}\right)}=0
$$

Combining all these cases we discover

$$
\int_{\mathfrak{L}_{l}} \chi_{\sigma_{j}}(\eta) d \eta=2 \pi i \delta_{l j}, \quad l, j=1,2, \ldots, n-1 .
$$

On substituting the integrals (3.32) and (3.37) into equations (3.26) we determine all the constants $a_{1}, a_{2}, \ldots, a_{n-1}$

$$
a_{j}=a_{0}+\frac{1}{2 \pi} \operatorname{Im} \sum_{l=0}^{n-1} \int_{\mathfrak{L}_{l}} \operatorname{Im}(c \eta) \chi_{\sigma_{j}}(\eta) d \eta, \quad j=1,2, \ldots, n-1 .
$$

The constant $a_{0}$ remains to be free. We thus proved that if the constants $a_{j}$ are chosen as in (3.38), then $\Phi_{1}(\zeta)$ is a $\mathfrak{G}$-automorphic function. Show finally that it satisfies the 
Riemann-Hilbert boundary condition (3.9). On splitting $\mathfrak{G}$ into $\sigma_{0}$ and $\mathfrak{G} \backslash \sigma_{0}$ we represent the function $\Psi_{1}(\zeta)$ in the form

$$
\begin{gathered}
\Psi_{1}(\zeta)=\frac{1}{2 \pi} \sum_{l=0}^{n-1} \int_{\mathfrak{L}_{l}}\left(\frac{1}{\eta-\zeta}-\frac{1}{\eta-\zeta_{*}}\right)\left[\operatorname{Im}(c \eta)-a_{l}\right] d \eta \\
+\frac{1}{2 \pi} \sum_{l=0}^{n-1} \sum_{\sigma \in \mathfrak{G} \backslash \sigma_{0}} \int_{\mathfrak{L}_{l}}\left(\frac{1}{\eta-\sigma(\zeta)}-\frac{1}{\eta-\sigma\left(\zeta_{*}\right)}\right)\left[\operatorname{Im}(c \eta)-a_{l}\right] d \eta .
\end{gathered}
$$

Passing to the limit $\zeta \rightarrow \xi \in \mathfrak{L}_{j}$ and employing the Sokhotski-Plemelj formulas we obtain

$$
\Psi_{1}^{-}(\xi)=-\frac{i}{2}\left[\operatorname{Im}(c \xi)-a_{j}\right]+\Psi_{1}(\xi)
$$

In a similar fashion we next analyze the function $\overline{\Psi_{1}\left(T_{0}(\zeta)\right)}$. We have

$$
\lim _{\zeta \rightarrow \xi \in \mathfrak{L}_{j}, \zeta \in \mathfrak{D}^{e}} \overline{\Psi_{1}\left(T_{0}(\zeta)\right)}=-\frac{i}{2}\left[\operatorname{Im}(c \xi)-a_{j}\right]+\overline{\Psi_{1}\left(T_{0}(\xi)\right)}
$$

and then the limit values $\Phi_{1}^{ \pm}(\xi)$ of the solution become

$$
\Phi_{1}^{ \pm}(\xi)= \pm i\left[\operatorname{Im}(c \xi)-a_{j}\right]+\Psi_{1}(\xi)+\overline{\Psi_{1}\left(T_{0}(\xi)\right)}, \quad \xi \in \mathfrak{L}_{j} .
$$

This verifies that the $\mathfrak{G}$-automorphic symmetric function $\Phi_{1}(\zeta)$ bounded at infinity and given by (3.19) with the constants $a_{j}$ defined by (3.38) solves the first Riemann-Hilbert problem (3.9). Any other solution of this problem differs from the function (3.19) by a constant. This may be proved in a manner standard in the theory of boundary value problems of the theory of analytic functions ([32).

\subsection{The function $\Phi_{2}(\zeta)$}

We begin with rewriting the second Riemann-Hilbert problem (3.9) in the following form:

$$
\Phi_{2}^{+}(\xi)-\Phi_{2}^{-}(\xi)=-2 i \tilde{d}_{j}+2 i g_{2 j}^{\circ}(\xi), \quad \zeta \in \mathfrak{L}_{j}, \quad j=0,1, \ldots, n-1,
$$

where

$$
\tilde{d}_{j}=\frac{\kappa_{j}}{\kappa_{j}-1} d_{j}^{\prime}, \quad g_{2 j}^{\circ}=\operatorname{Re}\left(\frac{\kappa_{j}}{\kappa_{j}-1} F(\xi)+\frac{\bar{\tau}}{\mu} c_{-1} \xi\right)
$$

and

$$
F(\xi)=c \xi-i\left[\operatorname{Im}(c \xi)-a_{j}\right]+\Psi_{1}(\xi)+\overline{\Psi_{1}\left(T_{0}(\xi)\right)}, \quad \xi \in \mathfrak{L}_{j} .
$$

The Riemann-Hilbert problem is solved in the same fashion as the first problem for the function $\Phi_{1}(\zeta)$. Its solution is given by

$$
\Phi_{2}(\zeta)=\Psi_{2}(\zeta)+\overline{\Psi_{2}\left(T_{0}(\zeta)\right)}+C_{2},
$$

where $C_{2}$ is an arbitrary real constant and

$$
\Psi_{2}(\zeta)=\frac{1}{2 \pi} \sum_{j=0}^{n-1} \int_{\mathfrak{L}_{j}}\left[g_{2 j}^{\circ}(\eta)-\tilde{d}_{j}\right] \mathcal{K}(\zeta, \eta) d \eta, \quad \zeta \notin \mathfrak{L} .
$$




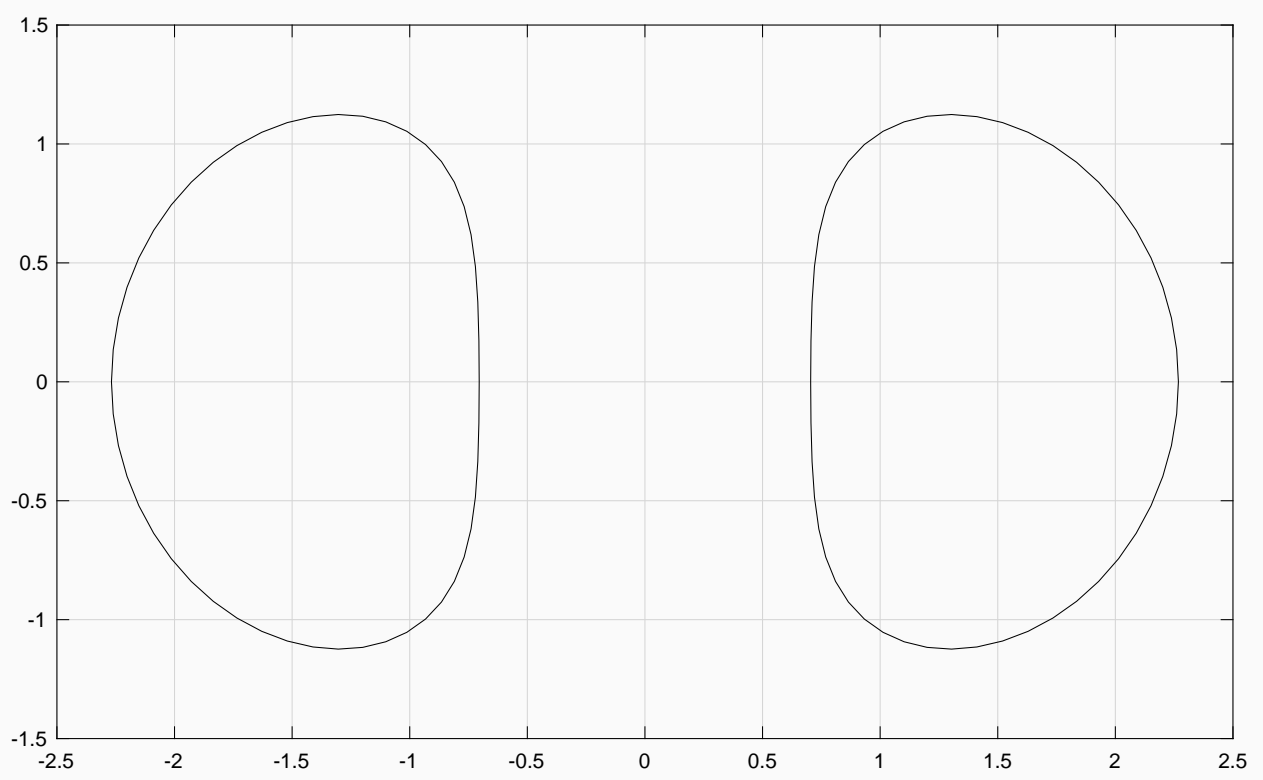

Figure 1: Two symmetric inclusions $(n=2)$ when $\tau_{1} / \mu=2, \tau_{1}^{\infty} / \mu=1, \tau_{2}=\tau_{2}^{\infty}=0$, $\kappa_{0}=\kappa_{1}=2, c_{-1}=1, r_{0}=r_{1}=1, \zeta_{0}=-1.5, \zeta_{1}=1.5, \zeta_{*}=0 . a_{0}=-a_{1}$, and $\tilde{d}_{0}=-\tilde{d}_{1}$.

This functions is a $\mathfrak{G}$-automorphic function if and only if the constants $\tilde{d}_{j}$ are given by

$$
\tilde{d}_{j}=\tilde{d}_{0}+\frac{1}{2 \pi} \operatorname{Im} \sum_{l=0}^{n-1} \int_{\mathfrak{L}_{l}} g_{2 l}^{\circ}(\eta) \chi_{\sigma_{j}}(\eta) d \eta, \quad j=1,2, \ldots, n-1 .
$$

The constant $\tilde{d}_{0}$ may be fixed arbitrarily. As before, it is directly verified that the function $\Phi_{2}(\xi)$ satisfies the symmetry condition (3.8). On the circles $\mathfrak{L}_{j}$, the limit values of the function $\Phi_{2}(\xi)$ are determined according to the Sokhotski-Plemelj formulas

$$
\Phi_{2}^{ \pm}(\xi)= \pm i\left[g_{2 j}^{\circ}(\xi)-\tilde{d}_{j}\right]+\Psi_{2}(\xi)+\overline{\Psi_{2}\left(T_{0}(\xi)\right)}, \quad \xi \in \mathfrak{L}_{j} .
$$

where $\Psi_{2}(\xi)$ and $\overline{\Psi_{2}\left(T_{0}(\xi)\right)}$ are the Cauchy principal values of the integrals $\Psi_{2}(\zeta)$ and $\overline{\Psi_{2}\left(T_{0}(\zeta)\right)}$, respectively, with $\zeta=\xi \in \mathfrak{L}_{j}$.

\section{Conformal mapping. Numerical results}

The conformal mapping $\omega(\zeta)$ can be expressed in terms of the solution of the second Riemann-Hilbert problem. From (3.5) and (3.49) we have

$$
\omega(\xi)=c_{-1} \xi-\frac{\mu}{\bar{\tau}}\left[g_{2 j}^{\circ}(\xi)-\tilde{d}_{j}+i \Psi_{2}(\xi)+i \overline{\Psi_{2}\left(T_{0}(\xi)\right)}\right], \quad \xi \in \mathfrak{L}_{j}, \quad j=0,1, \ldots, n-1 .
$$

When a point $\xi$ traverses the circle $\mathfrak{L}_{j}$, the point $z=\omega(\xi)$ describes the circumference of the inclusion $D_{j}(j=0,1, \ldots, n-1)$. Formula (4.1) found represents $(3 n-4)$-parametric family of conformal mappings of the $n$-connected circular domain into the $n$-connected physical domain. The free parameters of this family are the radii of the circles $\mathfrak{L}_{j}, r_{j}$, $(j=1,2, \ldots, n-1)$, the real center $\zeta_{1}$ of the circle $\mathfrak{L}_{1}$, and the complex centers $\zeta_{j}=\zeta_{j}^{\prime}+i \zeta_{j}^{\prime \prime}$ of the circles $\mathfrak{L}_{j}(j=2,3, \ldots, n-1)$. Up to transformations of translation, rotation, and scaling the conformal mapping is invariant with respect to the real constants $a_{0}$ and $\tilde{d}_{0}$, the complex parameter $c_{-1}$, and the point $\zeta_{*} \in \mathfrak{D}^{e}$. 

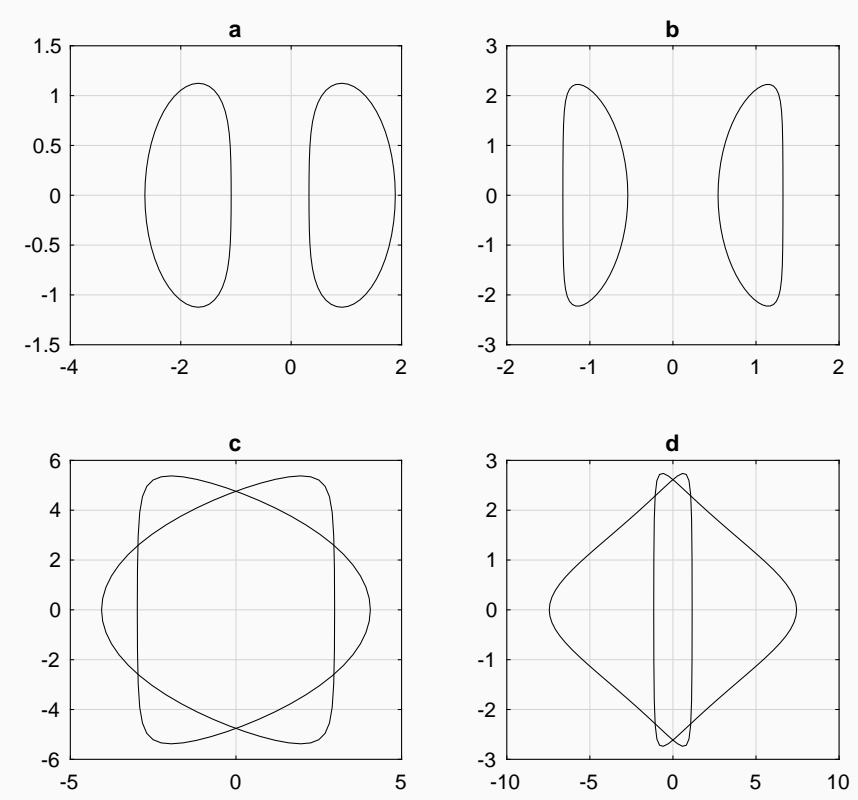

Figure 2: Samples of the contours $L_{0}$ and $L_{1}$ when $\tau_{1} / \mu=2, \tau_{1}^{\infty} / \mu=1, \tau_{2}=\tau_{2}^{\infty}=0$, $c_{-1}=1, r_{0}=r_{1}=1, \zeta_{0}=-1.5, \zeta_{1}=1.5$, and $\zeta_{*}=0$. a: $\kappa_{0}=\kappa_{1}=2, a_{0}=\tilde{d}_{0}=0$. b-d: $a_{0}=-a_{1}$, and $\tilde{d}_{0}=-\tilde{d}_{1}$. b: $\kappa_{0}=\kappa_{1}=0.5$. c: $\kappa_{0}=\kappa_{1}=0.9$. b: $\kappa_{0}=\kappa_{1}=1.1$.

To implement the method, one needs to compute the integrals $\Psi_{2}(\xi)$ and $\overline{\Psi_{2}\left(T_{0}(\xi)\right)}$. The former integral is

$$
\Psi_{2}(\xi)=\frac{1}{2 \pi} \sum_{j=0}^{n-1} \int_{\mathfrak{L}_{j}}\left[g_{2 j}^{\circ}(\eta)-\tilde{d}_{j}\right] \mathcal{K}(\xi, \eta) d \eta, \quad \xi \in \mathfrak{L}_{j}, \quad j=0,1, \ldots, n-1 .
$$

The kernel $\mathcal{K}(\xi, \eta)$ can be written in the form

$$
\begin{aligned}
\mathcal{K}(\xi, \eta)= & \frac{1}{\xi-\eta}-\frac{1}{\xi-\zeta_{*}}+\sum_{k_{1}=0}^{n-1} \sum_{k_{2}=0, k_{2} \neq k_{1}}^{n-1}\left(\frac{1}{\eta-T_{k_{1}} T_{k_{2}}(\xi)}-\frac{1}{\eta-T_{k_{1}} T_{k_{2}}\left(\zeta_{*}\right)}\right) \\
+ & \sum_{k_{1}=0}^{n-1} \sum_{k_{2}=0, k_{2} \neq k_{1}}^{n-1} \sum_{k_{3}=0, k_{3} \neq k_{2}}^{n-1} \sum_{k_{4}=0, k_{4} \neq k_{3}}^{n-1}\left(\frac{1}{\eta-T_{k_{1}} T_{k_{2}} T_{k_{3}} T_{k_{4}}(\xi)}\right. \\
& \left.-\frac{1}{\eta-T_{k_{1}} T_{k_{2}} T_{k_{3}} T_{k_{4}}\left(\zeta_{*}\right)}\right)+\ldots, \quad \xi \in \mathfrak{L}_{j}, \quad \eta \in \mathfrak{L}_{l} .
\end{aligned}
$$

Clearly, $\mathcal{K}(\xi, \eta)$ is a regular kernel if $j \neq l$, and a singular kernel otherwise. The singular part of the integral (4.2) is evaluated numerically by the formula

$$
\begin{gathered}
\frac{1}{2 \pi} \int_{\mathfrak{L}_{l}} \frac{\phi(\eta) d \eta}{\eta-\xi}=\frac{i}{2(2 N+1)} \sum_{j=-N}^{N} \phi\left(\zeta_{l}+r_{l} e^{i \theta_{j}}\right) \\
\times\left[1+\frac{2 i \sin \frac{N}{2}\left(\theta-\theta_{j}\right) \sin \frac{N+1}{2}\left(\theta-\theta_{j}\right)}{\sin \frac{\theta-\theta_{j}}{2}}\right], \quad \xi \in \mathfrak{L}_{l},
\end{gathered}
$$

where

$$
\theta_{j}=\frac{2 \pi j}{2 N+1}, \quad \theta=-i \ln \frac{\xi-\zeta_{l}}{r_{l}}
$$



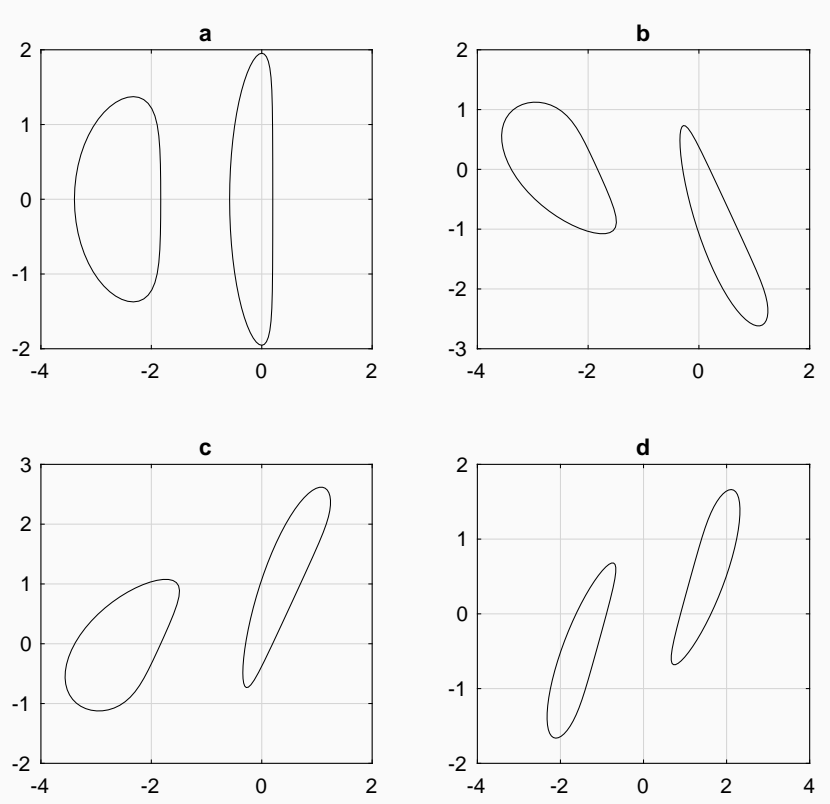

Figure 3: Samples of the contours $L_{0}$ and $L_{1}$ when $\tau_{1} / \mu=2, \tau_{1}^{\infty} / \mu=1, c_{-1}=1$, $r_{0}=r_{1}=1, \zeta_{0}=-1.5, \zeta_{1}=1.5, \zeta_{*}=0, a_{0}=-a_{1}$, and $\tilde{d}_{0}=-\tilde{d}_{1}$. a: $\tau_{2}=\tau_{2}^{\infty}=0$, $\kappa_{0}=2, \kappa_{1}=0.5$. b: $\tau_{2} / \mu=\tau_{2}^{\infty} / \mu=1, \kappa_{0}=2, \kappa_{1}=0.5$. c: $\tau_{2} / \mu=\tau_{2}^{\infty} / \mu=-1, \kappa_{0}=2$, $\kappa_{1}=0.5 . \mathrm{d}: \tau_{2} / \mu=\tau_{2}^{\infty} / \mu=-1, \kappa_{0}=\kappa_{1}=10$.

and $N$ is a sufficiently large positive integer.

For computing the function $\Psi_{2}\left(T_{0}(\xi)\right)$, we need the kernel $\mathcal{K}\left(T_{0}(\xi), \eta\right)$. Since $T_{0}(\xi)=$ $\xi, \xi \in \mathfrak{L}_{0}$, in the case $\xi \in \mathfrak{L}_{0}$ the kernel $\mathcal{K}\left(T_{0}(\xi), \eta\right)$ is given by formula (4.3). If $\xi \in \mathfrak{L}_{j}$, $j=1,2, \ldots, n-1$, and $j=l$, then since $\sigma_{j} T_{0}(\xi)=T_{j}(\xi)=\xi$, we have

$$
\begin{gathered}
\mathcal{K}\left(T_{0}(\xi), \eta\right)=\frac{1}{\eta-\xi}-\frac{1}{\eta-\sigma_{j}\left(\zeta_{*}\right)}+\frac{1}{\eta-T_{0}(\xi)}-\frac{1}{\eta-\zeta_{*}} \\
+\sum_{\sigma \in \mathfrak{G} \backslash \sigma_{0} \backslash \sigma_{j}}\left(\frac{1}{\eta-\sigma T_{0}(\xi)}-\frac{1}{\eta-\sigma\left(\zeta_{*}\right)}\right), \quad \xi \in \mathfrak{L}_{j} .
\end{gathered}
$$

The inverse model problem of antiplane elasticity solved has $n+4$ parameters, $\tau_{1} / \mu$, $\tau_{2} / \mu, \tau_{1}^{\infty} / \mu \tau_{2}^{\infty} / \mu$, and $\kappa_{j}(j=0,1, \ldots, n-1)$, The conformal mapping $z=\omega(\zeta)$ possesses $3 n+6$ real parameters, $\zeta_{j}=\zeta_{j}^{\prime}+i \zeta_{j}^{\prime \prime}, r_{j}(j=0,1, \ldots, n-1), c_{-1}=c_{-1}^{\prime}+i c_{-1}^{\prime \prime}, \zeta_{*}=\zeta_{*}^{\prime}+i \zeta_{*}^{\prime \prime}$, $a_{0}$, and $\tilde{d}_{0}$. Without loss of generality the ten real parameters $\zeta_{0}^{\prime}+i \zeta_{0}^{\prime \prime}, \zeta_{1}^{\prime \prime}, r_{0}, c_{-1}^{\prime}+i c_{-1}^{\prime \prime}$, $\zeta_{*}^{\prime}+i \zeta_{*}^{\prime \prime}, a_{0}$, and $\tilde{d}_{0}$ may be arbitrarily fixed. The other $3 n-4$ parameters are free and generate a family of uniformly stressed inclusions.

Figures 11 to 3 provides samples of two inclusions when the conformal map has two free parameters, the $x$-coordinate of the center and the radius of the circle $\mathfrak{L}_{1}$. In Fig. 1, we show the profiles of two symmetric inclusions in the case when the loading parameters are $\tau_{1} / \mu=2, \tau_{1}^{\infty} / \mu=1, \tau_{2}=\tau_{2}^{\infty}=0$, the materials of the inclusions and the matrix are characterized by the parameters $\kappa_{1}=2$ and $\kappa_{2}=2$. The circles $\mathfrak{L}_{1}$ and $\mathfrak{L}_{2}$ are taken to have unit radius, $r_{0}=r_{1}=1$, and centered at $\zeta_{0}=-1.5$ and $\zeta_{1}=1.5$, respectively. The point $\zeta_{*} \in \mathfrak{D}^{e}$ is chosen as the origin, $\zeta_{*}=0$. It turns out that when the parameters $\kappa_{1}$ and $\kappa_{2}$ tend to either 0 or infinity the contours $L_{0}$ and $L_{1}$ become slim and, in the limit, they become two segments. On the other hand, for $\kappa_{0}$ and $\kappa_{1}$ close to 1 , the contours $L_{0}$ and $L_{1}$ may intersect each other as shown in Fig. 2, Fig. 3 provides some examples of 


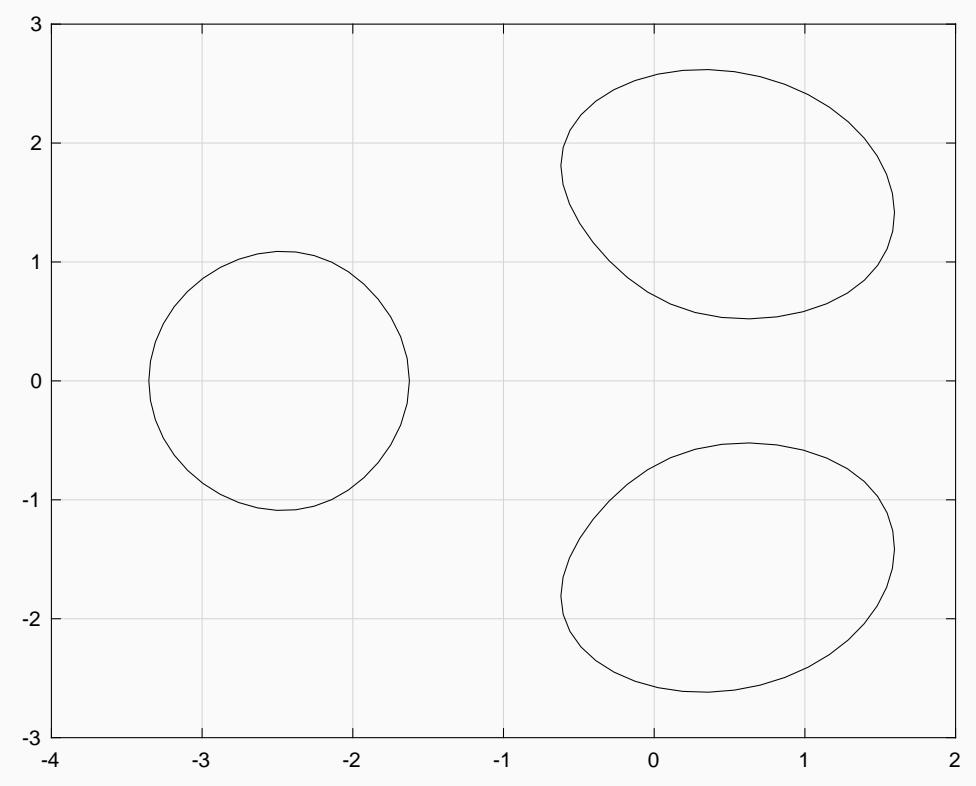

Figure 4: Three uniformly stressed inclusions when $\tau_{1} / \mu=2, \tau_{1}^{\infty} / \mu=1, \tau_{2}=\tau_{2}^{\infty}=0$, $\kappa_{0}=\kappa_{1}=\kappa_{2}=2, c_{-1}=1, r_{0}=r_{1}=r_{2}=1, \zeta_{0}=-2, \zeta_{1}=2 e^{\pi i / 3}, \zeta_{2}=2 e^{-\pi i / 3}, \zeta_{*}=0$, and $a_{0}=\tilde{d}_{0}=0$.

the problem and conformal map parameters which generate nonsymmetric contours $L_{0}$ and $L_{1}$.

In the case of three inclusions, the conformal map has five free parameters. Fig. 4 illustrates a symmetric case when $\tau_{1} / \mu=2, \tau_{1}^{\infty} / \mu=1, \tau_{2}=\tau_{2}^{\infty}=0$, the parameters $\kappa_{j}$ and the radii $r_{j}$ are the same: $\kappa_{0}=\kappa_{1}=\kappa_{2}=2, r_{0}=r_{1}=r_{2}=1$, and the circles centers are taken as $\zeta_{0}=-2, \zeta_{1}=2 e^{\pi i / 3}, \zeta_{2}=2 e^{-\pi i / 3}$. We give an example of nonsymmetric uniformly stressed inclusions in Fig. [5] when $\kappa_{0}=2, \kappa_{1}=3, \kappa_{2}=0.5$, $\tau_{1} / \mu=2, \tau_{1}^{\infty} / \mu=1, \tau_{2} / \mu=1, \tau_{2}^{\infty} / \mu=-1$. The radii and the centers of the circles $\mathfrak{L}_{j}$ are the same as in Fig. 4.

\section{Conclusion}

To solve the inverse problem of antiplane elasticity on recovering the shape of $n$ uniformly stressed inclusions, we proposed to apply the method of conformal mappings from an $n$-connected external circular domain to the exterior of $n$ inclusions. The reconstruction of the conformal map requires solving two Schwarz problems on $n$ circles when the right hand-side of the boundary condition of the second problem is expressed through the solution of the first Schwarz problem. To solve these Schwarz problems, we applied the method of symmetry and linear rational transformations. This approach brought us to two Riemann-Hilbert problems of the theory of automorphic functions generated by a Schottky group of symmetric linear rational transformations. By employing a quasiautomorphic analogue of the Cauchy kernel we derived a series representation of a family of conformal mappings meeting the requirements of the inverse problem of antiplane elasticity. This family possesses $3 n-4$ free real parameters which have to be chosen in a sensible manner to avoid possible overlapping of the inclusions.

Numerical results obtained for two- and three-connected domains revealed that when the parameters $\kappa_{j} \rightarrow 0$ or $\kappa_{j} \rightarrow \infty$, the inclusions tend to transform into segments. Here, 


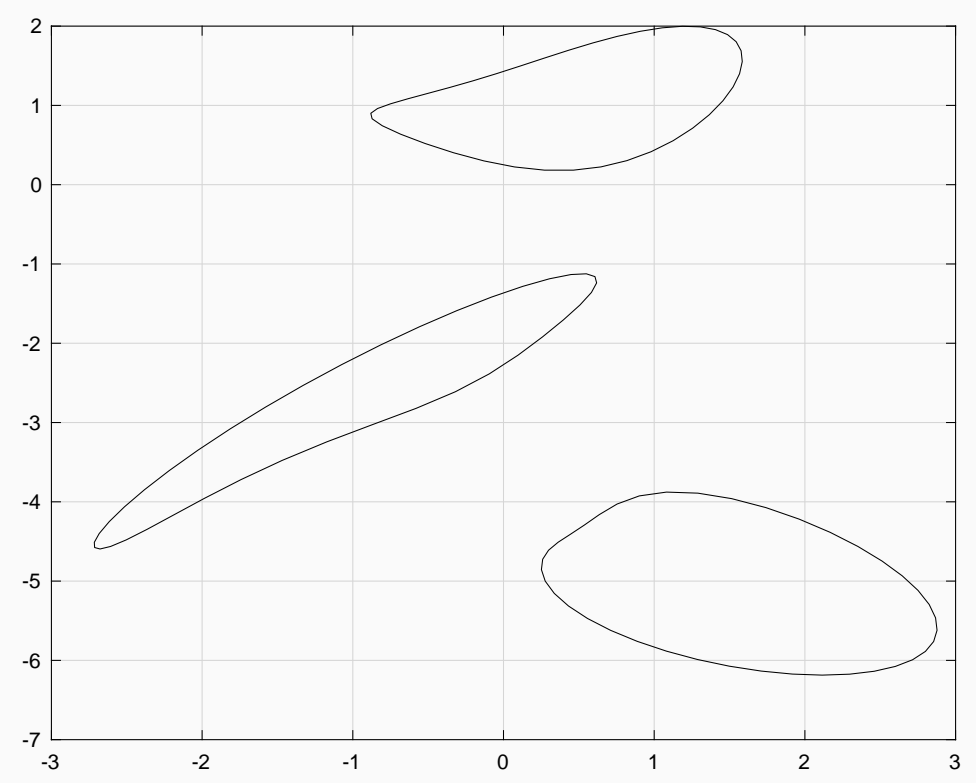

Figure 5: Three uniformly stressed inclusions when $\tau_{1} / \mu=2, \tau_{1}^{\infty} / \mu=1, \tau_{2} / \mu=1$, $\tau_{2}^{\infty} / \mu=-1, \kappa_{0}=2, \kappa_{1}=3, \kappa_{2}=0.5, c_{-1}=1, r_{0}=r_{1}=r_{2}=1, \zeta_{0}=-2, \zeta_{1}=2 e^{\pi i / 3}$, $\zeta_{2}=2 e^{-\pi i / 3}, \zeta_{*}=0$, and $a_{0}=\tilde{d}_{0}=0$.

$\kappa_{j}=\mu_{j} / \mu, \mu_{j}$ and $\mu$ are the shear moduli of the inclusions $D_{j}$ and the matrix $D^{e}$, and $j=0,1, \ldots, n-1$.

In this paper the preimage parametric domain was chosen to be the exterior of $n$ circles. Another possibility for the parametric domain is the exterior of $n$ slits lying in the same line (the real axis for example). In this case the slit map was constructed (33) by solving two Riemann-Hilbert problems on a symmetric genus- $n$ Riemann surface. An advantage of such an approach is its ability to recover the conformal map by quadratures in the cases of doubly and triply connected domains. However, when $n \geq 4$, since not each $n$-connected domain $D^{e}$ can be considered as an image by a slit map with $n$ slits lying in the same line, the method of slit maps is in general inapplicable. At the same time, if $n \geq 4$ and the associated Schottky group of the first class that is the series representation of the quasiautomorphic analogue of the Cauchy kernel is absolutely convergent, then the method of circular maps and the Riemann-Hilbert problems of the theory of automorphic functions works and gives a series representation of the conformal map. The set of domains associated with the first class Schottky group is broader and includes not only the set of $n$ circles whose centers fall in the same line.

\section{Appendix: Single inclusion}

Without loss of generality $\mathfrak{L}_{0}$ is the unit circle centered at the origin and $a_{0}=0$. The solution of the Schwarz problem (2.9), (2.11) for the unit circle $\mathfrak{L}_{0}$ is given by

$$
F(\zeta)=\beta_{0}-i \beta_{1} \zeta+i \bar{\beta}_{1} \zeta^{-1}
$$

where

$$
\beta_{1}=\beta_{1}^{\prime}+i \beta_{1}^{\prime \prime}=\frac{\bar{\tau}^{\infty}-\bar{\tau}}{\mu} i c_{-1}
$$


and $c_{-1}$ and $\beta_{0}$ are real constants. The solution of the second Schwarz problem (2.10), (2.11) can be represented in the form

$$
\bar{\tau} \omega(\zeta)=\gamma_{-1} \zeta^{-1}+\gamma_{0}+\gamma_{1} \zeta
$$

where $\gamma_{j}=\gamma_{j}^{\prime}+i \gamma_{j}^{\prime \prime}, j=-1,0,1$. On substituting the expressions (A.3) and (A.1) into the boundary condition (2.10) and replacing $\zeta$ by $e^{i \varphi}, 0 \leq \varphi \leq 2 \pi$, we derive

$$
\gamma_{0}^{\prime}=\left(\beta_{0}-d_{0}^{\prime}\right) \frac{\mu_{0}}{1-\kappa_{0}}, \quad \gamma_{-1}^{\prime}+\gamma_{1}^{\prime}=\frac{2 \beta_{1}^{\prime \prime} \mu_{0}}{1-\kappa_{0}}, \quad \gamma_{-1}^{\prime \prime}-\gamma_{1}^{\prime \prime}=\frac{2 \beta_{1}^{\prime} \mu_{0}}{1-\kappa_{0}} .
$$

Finally, by using the second formula in (2.11) and the relations (A.4) we determine the function $\omega(\zeta)$ up to an additive complex constant $\gamma$

$$
\omega(\zeta)=c_{-1}\left(\zeta+\frac{\delta}{\zeta}\right)+\gamma
$$

where

$$
\delta=\frac{2 \kappa_{0} \tau^{\infty}-\left(\kappa_{0}+1\right) \tau}{\left(1-\kappa_{0}\right) \bar{\tau}} .
$$

Let $\kappa_{0} \neq 1, \tau \neq 0$, and $\delta \neq \pm 1$. Then a point $z=\omega(\zeta)$ traces an ellipse $L_{0}$ whenever the point $\zeta$ traverses the unit circle $\mathfrak{L}_{0}$.

\section{References}

1. D. Riabuchinsky, Sur la détermination d'une surface d'aprés les données qu'elle porte, C.-R. Paris 189 (1929) 629-632.

2. L.A. Aksent'ev, N.B. Il'inskii, M.T. Nuzhin, R.B. Salimov and G.G. Tumashev, The theory of inverse boundary value problems for analytic functions and its applications, Mathematical Analysis, Akad. Nauk SSSR, Vsesoyuz. Inst. Nauchn. $i$ Tekhn. Informatsii Moscow 18 (1980) 67-124.

3. J.D. Eshelby, The determination of the elastic field of an ellipsoidal inclusion, and related problems, Proc. Roy. Soc. London A 241 (1957) 376-396.

4. G.P. Sendeckyj, Elastic inclusion problems in plane elastostatics, Int. J. Solids Structures 6 (1970 ) 1535-1543.

5. C.-Q. Ru and P. Schiavone, On the elliptic inclusion in anti-plane shear, Math. Mech. Solids 1 ( 1996) 327-333.

6. G.P. Cherepanov, Inverse problems of the plane theory of elasticity, J. Appl. Math. Mech. 38 ( 1974) 915-931.

7. S.B. Vigdergauz, Integral equation of the inverse problem of the plane theory of elasticity, J. Appl. Math. Mech. 40 (1976) 518-522.

8. Y. Grabovsky and R.V. Kohn, Microstructures minimizing the energy of a two phase elastic composite in two space dimensions. II.: The Vigdergauz microstructure, $J$. Mech. Phys. Solids 43 ( 1995 ) 949-972.

9. Y.A. Antipov, Slit maps in the study of equal-strength cavities in n-connected elastic planar domains, SIAM J. Appl. Math. 78 (2018) 320-342. 
10. H. Kang, E. Kim and G.W. Milton, Inclusion pairs satisfying Eshelby's uniformity property, SIAM, J. Appl. Math. 69 ( 2008) 577-595.

11. X. Wang, Uniform fields inside two non-elliptical inclusions, Math. Mech. Solids 17 (2012) 736-761.

12. L.P. Liu, Solutions to the Eshelby Conjectures, Proc. Roy. Soc. London A 464 ( 2008) 573-594.

13. M. Dai, C.-Q. Ru and C.-F. Gao, Uniform strain fields inside multiple inclusions in an elastic infinite plane under anti-plane shear, Math. Mech. Solids 17 (2017) 114-128.

14. Y.A. Antipov and V.V. Silvestrov, Method of Riemann surfaces in the study of supercavitating flow around two hydrofoils in a channel, Physica D 235 (2007) $72-81$.

15. Y.A. Antipov and V.V. Silvestrov, Circular map for supercavitating flow in a multiply connected domain, Quart. J. Mech. Appl. Math. 62 (2009) 167-200.

16. M.V. Keldysh, Conformal mappings of multiply connected domains on canonical domains, Uspekhi Matem. Nauk 6 (1939) 90-119.

17. R. Courant, Dirichlet's Principle, Conformal Mapping, and Minimal Surfaces (Interscience Publishers, Inc. New York 1950).

18. L.R. Ford, Automorphic Functions (McGraw-Hill Book Company, New York 1929).

19. L. I. Chibrikova and V. V. Silvestrov, On the question of the effectiveness of the solution of Riemann's boundary value problem for automorphic functions, Soviet Math. (Iz. VUZ) 12 (1978) 117-121.

20. V. V. Silvestrov, The Riemann boundary value problem for symmetric automorphic functions and its application, Theory of Functions of a Complex Variable and Boundary Value Problems (Chuvash. Gos. Univ., Cheboksary 1982) 93-107.

21. Y.A. Antipov and V.V. Silvestrov, Method of automorphic functions in the study of flow around a stack of porous cylinders, Quart. J. Mech. Appl. Math. 60 (2007) 337-366.

22. Y.A. Antipov and V.V. Silvestrov, Hilbert problem for a multiply connected circular domain and the analysis of the Hall effect in a plate, Quart. Appl. Math. 68 (2010) 563-590.

23. L. A. Aksent'ev, Construction of the Schwarz operator by the symmetry method, Trudy Sem. Kraev. Zadacham (Kazan) 4 (1967) 3-10.

24. I. A. Aleksandrov and A. S. Sorokin, The problem of Schwarz for multiply connected circular domains, Siberian Math. J. 13 (1973) 671-692.

25. V. V. Mityushev and S. V. Rogosin, Constructive Methods for Linear and Nonlinear Boundary Value Problems for Analytic Functions (Chapman \& Hall, Boca Raton 2000). 
26. A. Kazarin and Y. Obnosov, An exact analytical solution of an $R$-linear conjugation problem for a $n$-phased concentric circular heterogeneous structure, Appl. Math. Modeling 40 (2016) 5292-5300.

27. T.K. Delillo, A.R. Elcrat and J.A. Pfaltzgraff, Schwarz-Christoffel mappings of multiply connected domains, J. d'Analyse 94 (2004) 17-47.

28. D. Crowdy, The Schwarz-Christoffel mapping to multiply-connected polygonal domains, Proc. R. Soc. A 461 (2005) 2653-2678.

29. Y.A. Antipov and D.G. Crowdy, Riemann-Hilbert problem for automorphic functions and the Schottky-Klein prime function, Complex Anal. Oper. Theory 1 (2007) 317-334.

30. W. Burnside, On a class of automorphic functions, Proc. London Math. Soc. 23 (1892) 49-88.

31. F. Schottky, Ueber eine specielle Function, welche bei einer bestimmten linearen Transforma- tion ihres Arguments unverändert bleibt, J. Reine Angew. Math. 101 (1887) 227-272.

32. F. D. Gakhov, Boundary Value Problems (Pergamon Press, Oxford 1966).

33. Y.A. Antipov, Inverse antiplane problem on $n$ uniformly stressed inclusions, submitted for publication, arXiv:1705.06627 v2 . 